TRANSACTIONS OF THE

AMERICAN MATHEMATICAL SOCIETY

Volume 362, Number 7, July 2010, Pages 3377-3389

S 0002-9947(10)05142-1

Article electronically published on February 24, 2010

\title{
CONVEX VALUES AND LIPSCHITZ BEHAVIOR OF THE COMPLETE HULL MAPPING
}

\author{
J. P. MORENO
}

\begin{abstract}
This note continues the study initiated in 2006 by P.L. Papini, R. R. Phelps and the author on some classical notions from finite-dimensional convex geometry in spaces of continuous functions. Let $\mathcal{H}$ be the family of all closed, convex and bounded subsets of a Banach space endowed with the Hausdorff metric. A completion of $A \in \mathcal{H}$ is a diametrically maximal set $D \in \mathcal{H}$ satisfying $A \subset D$ and $\operatorname{diam} A=\operatorname{diam} D$. The complete hull mapping associates with every $A \in \mathcal{H}$ the family $\gamma(A)$ of all its possible completions. It is shown that the set-valued mapping $\gamma$ need not be convex valued even in finite-dimensional spaces, while, in the case of $C(K)$ spaces, $\gamma$ is convex valued if and only if $K$ is extremally disconnected. Regarding the continuity we prove that, again in $C(K)$ spaces, $\gamma$ is always Lipschitz continuous with constant less than or equal to 5 and has a Lipschitz selection with constant less than or equal to 3 . If we consider the analogous problem in Euclidean spaces, we show that $\gamma$ is Hölder continuous of order $1 / 4$ and locally Hölder continuous of order $1 / 2$, the Hölder constants depending on the diameter of the sets in both cases.
\end{abstract}

\section{INTRODUCTION}

The study of planar constant width sets can be traced back to Euler, who called them orbiformes 7, although they have been known also as spheroforms (in dimension three), spheroids, gleichdicke and sets of constant breadth. Lebesgue, Blaschke and Besicovitch are some of the many mathematicians that were attracted in the past by this classical subject in convexity. Most books devoted to this general area discuss at least some of their fascinating properties, which include curious industrial applications. A finite-dimensional bounded closed convex set $C$ has constant width $\lambda$ if the distance between any two parallel supporting hyperplanes of $C$ equals $\lambda$. As a counterpart of this concept, Meissner [12] introduced at the beginning of the last century the notion of a diametrically maximal set, which also appears in the literature under different names such as complete, diametrically complete and entire. A closed, bounded and convex set $C$ in a normed space is diametrically maximal if

$$
\operatorname{diam} C<\operatorname{diam}(C \cup\{x\})
$$

for every $x \notin C$. Sets of constant width are always diametrically maximal, but the converse, which is true in two-dimensional spaces and also in (finite-dimensional)

Received by the editors July 31, 2007.

2010 Mathematics Subject Classification. Primary 46E15, 52A05.

Key words and phrases. Diametrically maximal set, constant width set, complete hull mapping, $C(K)$ space.

This work was partially supported by the DGICYT project MTM 2006-03531.

(C)2010 American Mathematical Society 3377

Reverts to public domain 28 years from publication 
Euclidean spaces, is false in general. Both families of sets have been studied in several papers, mainly in the finite-dimensional case (see, for instance, the more than 260 items cited in the authoritative survey by Chakerian and Groemer [5]; a more recent account is contained in the survey by Heil and Martini [10]). The investigation of constant width sets in the infinite-dimensional setting began with the pioneering work of Berhends and Harmand [2]. Berhends [3, Payá and RodríguezPalacios [18, and the latter author [19], 20] pursued the study of this subject that has been recently continued by Papini, Phelps and the author in 15. Diametrically maximal sets in general Banach spaces appeared, in connection with the Jung constant, in a paper by Franchetti [8, while Baronti and Papini later carried out a systematic study of this topic [1]. Finally, some new families of convex sets which are related to diametrically maximal and constant width sets have been introduced and studied in [16.

We will denote by $\mathcal{H}$ the family of all closed, convex and bounded sets endowed with the Hausdorff metric and by $\mathcal{D} \mathcal{M}$ the family of all diametrically maximal sets. The complete hull mapping $\gamma$ associates with each $C \in \mathcal{H}$ the family of all its possible completions, namely

$$
\gamma(C)=\{D \in \mathcal{D} \mathcal{M}: C \subset D, \operatorname{diam} D=\operatorname{diam} C\},
$$

and our starting point is the previous paper [14] where this mapping was investigated in the context of $C(K)$ spaces in connection with the concept of porosity. Roughly speaking it was proved there that, when $K$ is not a singleton, $\gamma$ is typically a multivalued mapping. A first step in order to investigate other basic properties of $\gamma$ is to know whether a useful description of $\gamma(C)$, for any $C \in \mathcal{H}$, is available. The difficulties in achieving such a description can be illustrated by the recent paper by T. Lachand-Robert and E. Oudet [11, where an explicit construction of constant width sets in any finite-dimensional Euclidean space is provided. The situation becomes more clear when dealing with $C(K)$ spaces; a characterization of $\gamma(C)$ was given in 14, the key ingredients being the use of some fine properties of semicontinuous functions. The class of $C(K)$ spaces plays an important role in several areas of mathematics, such as functional and real or complex analysis, infinite-dimensional topology and differential equations, and it has received constant attention in recent decades.

Some properties of the complete hull mapping are trivial. For instance, $\gamma(C)$ is bounded, for any $C \in \mathcal{H}$, and $\operatorname{diam} \gamma(C) \leq 2 \operatorname{diam} C$. Indeed, given $D, E \in \gamma(C)$, $\operatorname{dist}(D, E) \leq \operatorname{dist}(D, C)+\operatorname{dist}(C, E) \leq 2 \operatorname{diam} C$. However, some other properties are not so easy to check. For instance, is $\gamma(C)$ always closed? The answer is affirmative in finite-dimensional spaces and $C(K)$ spaces since, in these cases, $\mathcal{D} \mathcal{M}$ is closed [16. Then, if we consider $C \in \mathcal{H}$ and a sequence $\left\{C_{n}\right\}_{n} \subset \gamma(C)$ which converges to $D \in \mathcal{H}$, we know that $D \in \mathcal{D} \mathcal{M}$. Therefore, to prove that $D \in \gamma(C)$ we only need to take care of two details: $C \subset D$ and $\operatorname{diam} D=\operatorname{diam} C$. On the one hand,

$$
C \subset C_{n} \subset D+\left(\operatorname{dist}\left(C_{n}, D\right)+\frac{1}{n}\right) B
$$

for every $n \in \mathbb{N}$, where $B$ denotes the unit ball, and therefore $C \subset D$. On the other hand,

$$
\begin{aligned}
\operatorname{diam} C & =\operatorname{diam} C_{n} \\
& \leq \operatorname{diam} D+2 \operatorname{dist}\left(D, C_{n}\right) \leq \operatorname{diam} C_{n}+4 \operatorname{dist}\left(D, C_{n}\right) \\
& =\operatorname{diam} C+4 \operatorname{dist}\left(D, C_{n}\right)
\end{aligned}
$$


for every $n \in \mathbb{N}$, which shows that $\operatorname{diam} D=\operatorname{diam} C$. The questions of whether $\mathcal{D} \mathcal{M}$ is a closed family and whether $\gamma(C)$ is closed, for every $C \in \mathcal{H}$, seem to be open problems.

In this paper we deal with two questions: when is $\gamma$ convex valued (namely when is $\gamma(C)$ a convex family, for every $C \in \mathcal{H}$ ) and when is $\gamma$ continuous. In Section 2 we show that there are tridimensional spaces where $\gamma$ is not convex valued and prove a characterization for extremally disconnected compact Hausdorff spaces in terms of the convexity of the values of $\gamma$ in $C(K)$. In Section 3 we prove that, in $C(K), \gamma$ is Lipschitz continuous and has a Lipschitz selection. In Section 4 we show that, in Euclidean spaces, $\gamma$ is Hölder continuous of order $1 / 4$ and locally Hölder continuous of order $1 / 2$, the Hölder constants depending, in both cases, on the diameter of the sets. In particular, if $C, D \in \mathcal{H}$ have diameters $d_{C}, d_{D}$, respectively, and $\operatorname{dist}(C, D)<\varepsilon \leq 0.3 \min \left\{d_{C}, d_{D}\right\}$, then

$$
\operatorname{dist}(\gamma(C), \gamma(D)) \leq \max \left\{2,42 \varepsilon, \varepsilon+2,22 \sqrt{d_{C D}+2 \varepsilon} \sqrt{\varepsilon}\right\}
$$

where $d_{C D}=\max \left\{d_{C}, d_{D}\right\}$.

\section{When IS $\gamma$ CONVEX VALUED?}

We will first present some examples of finite-dimensional spaces where $\gamma$ is convex valued and then a tridimensional space where $\gamma$ fails to be convex valued. In these examples, the underlying idea is that Minkowski addition preserves the property of having constant width, while it does not preserve the property of being diametrically maximal, as proved in [15].

It should not come as a surprise that $\gamma$ is convex valued in Euclidean spaces: if $C \in \mathcal{H}$ and $D, F \in \gamma(C)$, then $\lambda D+(1-\lambda) F \in \gamma(C)$, for every $\lambda \in[0,1]$. Indeed, $C$ and $D$ are sets of constant width, and so $\lambda D+(1-\lambda) F \in \gamma(C)$ (recall that Minkowski addition preserves the property of having constant width, while it does not preserve the property of being diametrically maximal [15]). Obviously $C \subset \lambda D+(1-\lambda) F$, which implies $\operatorname{diam}(\lambda D+(1-\lambda) F) \geq \operatorname{diam} C$, and

$$
\operatorname{diam}(\lambda D+(1-\lambda) F) \leq \lambda \operatorname{diam} D+(1-\lambda) \operatorname{diam} F=\operatorname{diam} C .
$$

Beside this example, we have a finite-dimensional polyhedral space where $\gamma$ need not be convex valued: $\ell_{1}^{3}$. Consider the set $C=\operatorname{conv}\{(-1,-1,-1),(0,0,-1)\}$ and the two completions

$$
\begin{aligned}
D & =\operatorname{conv}\{C,(-1,0,0),(0,-1,0)\}, \\
D^{\prime} & =\operatorname{conv}\{C,(0,-1,-2),(-1,0,-2)\} .
\end{aligned}
$$

It is not difficult to prove that $D+D^{\prime} \notin \gamma(C)$ since, actually, $D+D^{\prime}$ is not an intersection of balls (see, e.g., Proposition 5.2 in 15]). One can now be tempted to think that $\gamma$ is not convex valued in $\ell_{1}^{3}$ simply because it is a polyhedral space. However, there is an easy example of a finite-dimensional polyhedral space where $\gamma$ is convex valued: $\ell_{\infty}^{3}$, where the only diametrically maximal sets are closed balls. Since $\ell_{\infty}^{3}$ is a particular case of a space of continuous functions, it is natural to ask whether $\gamma$ is always convex valued in $C(K)$, for every compact Hausdorff space $K$. We will see that this is not so, either. As a matter of fact, this question is used in Theorem 2.1 to characterize extremal disconnectedness.

Recall that a topological space is said to be extremally disconnected if every open set has an open closure. If $K$ is any compact Hausdorff space, the space $C(K)$ consists of all real-valued continuous functions $f: K \rightarrow \mathbb{R}$ under the sup 
norm $\|f\|=\max _{t \in K}|f(t)|$. A closed, convex and bounded set $C \subset C(K)$ is a nonempty intersection of balls if and only if there are $f, g: K \rightarrow \mathbb{R}$ lower and upper semicontinuous functions, respectively, such that $C=[f, g]=\{h \in C(K): f(t) \leq$ $h(t) \leq g(t), t \in K\}[13$.

Theorem 2.1. The complete hull mapping in $C(K)$ is convex valued if and only if $K$ is extremally disconnected.

Proof. To prove necessity, consider a closed, convex and bounded set $C \subset C(K)$ with $\operatorname{diam} C=d$, two completions $D, E \in \gamma(C)$ and, finally, a real number $0 \leq$ $\lambda \leq 1$. Since $K$ is extremally disconnected, there exist four continuous functions $f, g, f^{\prime}, g^{\prime}: K \rightarrow \mathbb{R}$ satisfying

$$
D=[f, g]=\{h \in C(K): f(t) \leq h(t) \leq g(t), t \in K\}
$$

and, analogously, $E=\left[f^{\prime}, g^{\prime}\right]$ (see, for instance, the proof of Theorem 3.4 in [13). Moreover, $f(t)-g(t)=f^{\prime}(t)-g^{\prime}(t)=d$ for every $t \in K$, since $D, E \in \gamma(C)$. Now, what about $\lambda D+(1-\lambda) E$ ? The condition $C \subset \lambda D+(1-\lambda) E$ is trivially fulfilled, so we only must check that $\lambda D+(1-\lambda) E$ is diametrically maximal and that $\operatorname{diam}(\lambda D+(1-\lambda) E)=d$. As a consequence of the Riesz decomposition property, we have

$$
\lambda D+(1-\lambda) E=\left[\lambda f+(1-\lambda) f^{\prime}, \lambda g+(1-\lambda) g^{\prime}\right],
$$

which proves the two needed conditions since

$$
\begin{aligned}
\lambda g(t) & +(1-\lambda) g^{\prime}(t)-\left(\lambda f(t)+(1-\lambda) f^{\prime}(t)\right) \\
& =\lambda(g(t)-f(t))+(1-\lambda)\left(g^{\prime}(t)-f^{\prime}(t)\right) \\
& =\lambda d+(1-\lambda) d=d
\end{aligned}
$$

for every $t \in K$.

To prove sufficiency, assume that there is an open set $G \subset K$ such that $\bar{G}$ is not open; we will find a closed, convex and bounded $C \subset C(K)$ for which $\gamma(C)$ is not convex. First of all, as $\bar{G}$ is not an open set, it is clear that $\bar{G} \neq K$ and hence there exists $t_{0} \in K \backslash \bar{G}$. Since $K$ is Hausdorff, we can find an open set $F \subset K$ such that $t_{0} \in F$ and $\bar{F} \cap \bar{G}=\emptyset$. We now define $f, g \in C(K)$ as follows: $g$ is the constant function $g(t)=1$, and $f$ is any Urysohn function with values in $[-1,0]$ satisfying $f\left(t_{0}\right)=-1$ and $f(t)=0$ for every $t \notin F$. Finally, we choose $C=[f, g]$, and we claim that $\gamma(C)$ is not convex. To prove the claim, consider the two lower semicontinuous functions

$$
f_{1}(t)=\left\{\begin{array}{ll}
-1 & \text { if } t \in K \backslash G, \\
0 & \text { if } t \in G,
\end{array} \quad f_{2}(t)= \begin{cases}0 & \text { if } \quad t \in K \backslash \bar{G} \cup \bar{F}, \\
-1 & \text { if } t \in \bar{G} \cup \bar{F}\end{cases}\right.
$$

and the corresponding convex subsets $D=\left[f_{1}, \check{f}_{1}+2\right]$ and $E=\left[f_{2}, \check{f}_{2}+2\right]$, where $\check{f}_{1}$ and $\check{f}_{2}$ are the upper semicontinuous envelopes of $f_{1}$ and $f_{2}$, respectively. The proof will be accomplished by showing that $D, E \in \gamma(C)$ but $\frac{1}{2}(D+E)$ is not an intersection of closed balls, thus implying that $\frac{1}{2}(D+E) \notin \gamma(C)$. The first part is easy since both $D$ and $E$ are diametrically maximal sets, both containing $C$, and $\operatorname{diam} D=\operatorname{diam} E=2=\operatorname{diam} C$. Suppose that $\frac{1}{2}(D+E)$ is an intersection of closed balls. Then there exist $\tilde{f}, \tilde{g}: K \rightarrow \mathbb{R}$ lower and upper semicontinuous functions, respectively, satisfying

$$
\frac{1}{2}(D+E)=[\tilde{f}, \tilde{g}]
$$


Since $C \subset \frac{1}{2}(D+E)$, it is clear that $\tilde{g}(t) \geq 1$ for every $t \in K$. On the other hand, $\inf \{h(t): h \in D\} \leq 0$ for every $t \in K$, and the same inequality holds replacing $D$ by $E$. Moreover, $\inf \{h(t): h \in D\}=-1$ when $t \in K \backslash \bar{G}$ and $\inf \{h(t): h \in E\}=-1$ when $t \in G$. As a consequence, $\inf \left\{h(t): h \in \frac{1}{2}(D+E)\right\} \leq-\frac{1}{2}$ when $t \in G \cup(K \backslash \bar{G})$. Taking into account that $\tilde{f}$ is lower semicontinuous and $G \cup(K \backslash \bar{G})$ is dense in $K$, we conclude that $\tilde{f}(t) \leq-\frac{1}{2}$ for every $t \in K$. The above considerations show that

$$
\frac{1}{2} B \subset \frac{1}{2}(D+E),
$$

where $B$ is the unit ball. In order to see how (2.1) leads to a contradiction, consider the set

$$
S=(\bar{G} \backslash G) \cap(\overline{K \backslash \bar{G}})
$$

which is nonempty because $\bar{G}$ is not open. Also, having in mind that $\bar{G} \cap \bar{F}=\emptyset$, it is clear that

$$
S=S \cap(\overline{K \backslash \bar{F}})=(\bar{G} \backslash G) \cap(\overline{K \backslash(\bar{G} \cup \bar{F})}),
$$

which, together with the definitions of $D$ and $E$, implies that $h(t) \geq 0$ for every $h \in \frac{1}{2}(D+E)$ and every $t \in S$, providing the desired contradiction to (2.1).

\section{Continuity of $\gamma$ in $C(K)$}

This section is devoted to studying the continuity and Lipschitz continuity of the complete hull mapping $\gamma$ in $C(K)$. The notion of continuity will be understood with respect to the Hausdorff metric both in $\mathcal{H}$ and in the hyperspace of closed subsets of $\mathcal{H}$, where $\gamma$ has its values. In particular, we will say that $\gamma$ is Lipschitz continuous if there is $\alpha \geq 0$ such that for every $C, D \in \mathcal{H}$, every $E \in \gamma(C)$ and every $F \in \gamma(D)$, there is $E^{\prime} \in \gamma(D)$ and $F^{\prime} \in \gamma(C)$ satisfying

$$
\max \left\{\operatorname{dist}\left(F, F^{\prime}\right), \operatorname{dist}\left(E, E^{\prime}\right)\right\} \leq \alpha \operatorname{dist}(C, D) .
$$

As usual, the infimum of such $\alpha$ is called the Lipschitz constant of the function $\gamma$. To prove the Lipschitz continuity of $\gamma$ we need a suitable description of $\gamma(C)$, for any $C \in \mathcal{H}$. We first consider its ball convex hull $\beta(C)$, namely the intersection of all closed balls containing $C$. The reason is that $\beta(C)$ has an easy representation, while this is not often the case of $C$, and $\gamma(C)=\gamma(\beta(C))$. Indeed,

$$
\beta(C)=\left[f_{C}, g_{C}\right]=\left[\hat{f}_{C}, \check{g}_{C}\right],
$$

where $f_{C}(t)=\inf \{h(t): h \in C\}, g_{C}(t)=\sup \{h(t): h \in C\}$ and $\hat{f}_{C}, \check{g}_{C}$ are the corresponding lower and upper semicontinuous envelopes of $f_{C}$ and $g_{C}$, respectively. The diameter of $C$ will be denoted by $\operatorname{diam} C$ or simply by $d_{C}$.

Theorem 3.1. In $C(K)$ spaces, the complete hull mapping $\gamma$ is Lipschitz and admits a Lipschitz selection.

Proof. Given $C \in \mathcal{H}$ and using the notation introduced above, define $\Gamma(C)$ as the family of those lower semicontinuous functions $\varphi: K \rightarrow \mathbb{R}$ satisfying

$$
\check{g}_{C}(t)-d_{C} \leq \varphi(t) \leq \hat{f}_{C}(t)
$$

for every $t \in \mathcal{D}_{\hat{f}_{C}} \cap \mathcal{D}_{\check{g}_{C}}$, where $\mathcal{D}_{\hat{f}_{C}}$ and $\mathcal{D}_{\check{g}_{C}}$ are the points of continuity of $\hat{f}_{C}$ and $\check{g}_{C}$, respectively. It is known [14 that

$$
\gamma(C)=\left\{\left[\varphi, \check{\varphi}+d_{C}\right]: \varphi \in \Gamma(C)\right\}
$$


which is the suitable representation that we need to prove the theorem. Consider $C, D \in \mathcal{H}$ and denote it by $\varepsilon=\operatorname{dist}(C, D)$. As a first step in the proof of the Lipschitz selection, we will see that

$$
\sup _{t \in K}\left|\hat{f}_{C}(t)-\hat{f}_{D}(t)\right| \leq \varepsilon
$$

and

$$
\sup _{t \in K}\left|\check{g}_{C}(t)-\check{g}_{D}(t)\right| \leq \varepsilon .
$$

Since $\operatorname{dist}(C, D) \leq \varepsilon$, for every $h \in C$ there is $h^{\prime} \in D$ satisfying

$$
\sup _{t \in K}\left|h(t)-h^{\prime}(t)\right| \leq \varepsilon,
$$

and the same is true when replacing $C$ by $D$. Then, it is clear that

$$
\sup _{t \in K}\left|f_{C}(t)-f_{D}(t)\right| \leq \varepsilon,
$$

and this implies (3.1). A similar argument can be used to prove (3.2). Now, if we prove that

$$
\operatorname{dist}\left(\left[\hat{f}_{C}, f_{C}+d_{C}\right],\left[\hat{f}_{D}, f_{D}+d_{D}\right]\right) \leq 3 \varepsilon
$$

then $C \rightarrow\left[\hat{f}_{C}, f_{C}+d_{C}\right]$ is the Lipschitz selection we are looking for (notice that $f_{C}$ is upper semicontinuous since it is an infimum of continuous functions, and so $\hat{f}_{C}=f_{C}$ ). The proof of the above inequality will follow from (3.1), provided we are able to show that

$$
\operatorname{dist}\left(\left[\hat{f}_{C}, f_{C}+d_{C}\right],\left[\hat{f}_{D}, f_{D}+d_{D}\right]\right) \leq \sup _{t \in \mathcal{D}}\left|\hat{f}_{C}(t)-\hat{f}_{D}(t)\right|+\left|d_{C}-d_{D}\right|,
$$

where $\mathcal{D}$ is any dense set contained in $\mathcal{D}_{\hat{f}_{C}} \cap \mathcal{D}_{\check{g}_{C}}$, $\operatorname{since} \sup _{t \in \mathcal{D}}\left|\hat{f}_{C}(t)-\hat{f}_{D}(t)\right| \leq \varepsilon$ and $\left|d_{C}-d_{D}\right| \leq 2 \varepsilon$. Denote this by $\tau=\sup _{t \in \mathcal{D}}\left|\hat{f}_{C}(t)-\hat{f}_{D}(t)\right|$. Since

$$
\hat{f}_{D}(t)-\tau \leq \hat{f}_{C}(t) \leq \hat{f}_{D}(t)+\tau
$$

for every $t \in \mathcal{D}$, the above inequalities hold for every $t \in \mathcal{D}_{\hat{f}_{C}} \cap \mathcal{D}_{\check{g}_{C}}$, because $\mathcal{D}$ is dense in $\mathcal{D}_{\hat{f}_{C}} \cap \mathcal{D}_{\check{g}_{C}}$. As a a consequence we get

$$
\begin{aligned}
{\left[\hat{f}_{C}, f_{C}+d_{C}\right] } & \subset\left[\hat{f}_{D}-\tau, f_{D}+d_{C}+\tau\right] \\
& \subset\left[\hat{f}_{D}-\tau-\left|d_{C}-d_{D}\right|, f_{D}+d_{D}+\tau+\left|d_{C}-d_{D}\right|\right] \\
& =\left[\hat{f}_{D}, f_{D}+d_{D}\right]+\left(\tau+\left|d_{C}-d_{D}\right|\right) B,
\end{aligned}
$$

where $B$ is the unit ball. Analogously,

$$
\left[\hat{f}_{D}, f_{D}+d_{D}\right] \subset\left[\hat{f}_{C}, f_{C}+d_{C}\right]+\left(\tau+\left|d_{C}-d_{D}\right|\right) B
$$

and hence $\operatorname{dist}\left(\left[\hat{f}_{C}, f_{C}+d_{C}\right],\left[\hat{f}_{D}, f_{D}+d_{D}\right]\right) \leq \tau+\left|d_{C}-d_{D}\right|$, showing (3.4) and the existence of the continuous selection. Before starting with the question of the continuity of $\gamma$, let us mention that (3.4) can be completed with the following estimate from below:

$$
\sup _{t \in \mathcal{D}}\left|\hat{f}_{C}(t)-\hat{f}_{D}(t)\right| \leq \operatorname{dist}\left(\left[\hat{f}_{C}, f_{C}+d_{C}\right],\left[\hat{f}_{D}, f_{D}+d_{D}\right]\right) .
$$

To prove the above inequality, fix an arbitrary $\delta>0$ and choose $s \in \mathcal{D}$ satisfying $\left|\hat{f}_{C}(s)-\hat{f}_{D}(s)\right| \geq \tau-\delta / 2$. Suppose, for instance, that $\left|\hat{f}_{C}(s)-\hat{f}_{D}(s)\right|=\hat{f}_{C}(s)-\hat{f}_{D}(s)$ (the other case is analogous) and pick $\varphi \in D$ such that $\varphi(s)<\hat{f}_{D}(s)+\delta / 2$ (the 
existence of such $\varphi$ follows from Proposition 3.3 in [13]). Then, for every $h \in C$, we have

$$
h(s)-\varphi(s) \geq \hat{f}_{C}(s)-\hat{f}_{D}(s)-\delta / 2 \geq \tau-\delta
$$

and

$$
\operatorname{dist}\left(\left[\hat{f}_{C}, f_{C}+d_{C}\right],\left[\hat{f}_{D}, f_{D}+d_{D}\right]\right) \geq \operatorname{dist}\left(\left[\hat{f}_{C}, f_{C}+d_{C}\right], \varphi\right) \geq \tau-\delta ;
$$

hence (3.5) is proved. The proof of the first part of the theorem relies on the following two general claims.

Claim 1. When a diametrically maximal set $A$ contains $C \in \mathcal{H}$, it also contains a completion of $C$. Indeed, there are $f, g: K \rightarrow \mathbb{R}$ lower and upper semicontinuous functions, respectively, such that $A=[f, g]$ and $g(t)-f(t)=\operatorname{diam} A$ for every $t \in \mathcal{D}_{f} \cap \mathcal{D}_{g}$. Since $A$ is an intersection of balls, it is clear that

$$
\left[\hat{f}_{C}, \check{g}_{C}\right] \subset[f, g]
$$

and, obviously, $d_{C}=\operatorname{diam} C \leq \operatorname{diam} A$. Define

$$
f^{\prime}=\max \left\{f, g_{C}-d_{C}\right\},
$$

which is a lower semicontinuous function satisfying $f \leq f^{\prime}$. Now consider $g_{0}=$ $f^{\prime}+d_{C}$ and its corresponding upper semicontinuous envelope $\check{g_{0}}=g^{\prime}$. We define, finally,

$$
H=\left[f^{\prime}, g^{\prime}\right]
$$

and check that $H \subset A$ and $H \in \gamma(C)$. The first part is straightforward, since $f \leq f^{\prime}$ and

$$
g_{0}=\max \left\{f+d_{C}, g_{C}\right\} \leq g,
$$

so consequently $g^{\prime} \leq g$ also, and so $\left[f^{\prime}, g^{\prime}\right] \subset[f, g]$. For the second part, notice that $g^{\prime}(t)-f^{\prime}(t)=d_{C}$ for every $t \in \mathcal{D}_{g_{0}}=\mathcal{D}_{f}$, which is a dense subset of $\mathcal{D}_{g^{\prime}}$, so by a continuity argument, $g^{\prime}(t)-f^{\prime}(t)=d_{C}$ for every $t \in \mathcal{D}_{g^{\prime}} \cap \mathcal{D}_{f^{\prime}}$, thus implying that $H$ is diametrically maximal and has diameter $d_{C}$. Finally we need to prove that

$$
\left[\hat{f}_{C}, \check{g}_{C}\right] \subset\left[f^{\prime}, g^{\prime}\right] \text {. }
$$

On the one hand, since $g_{0}=\max \left\{f+d_{C}, g_{C}\right\}$, we have $g_{C} \leq g_{0}$ and, taking upper semicontinuous envelopes, we obtain $\check{g}_{C} \leq g^{\prime}$. On the other hand, it is clear that $g_{C}(t)-f_{C}(t) \leq d_{C}$ for every $t \in K$, and also $f_{C} \geq f$, because $C \subset[f, g]$, hence $f_{C} \geq f^{\prime}$. Since $f^{\prime}$ is lower semicontinuous, this implies $\hat{f}_{C} \geq f^{\prime}$, thus proving (3.6).

Claim 2. If $A, H \in \mathcal{H}$ are two diametrically maximal sets such that $H \subset A$, then $\operatorname{dist}(A, H) \leq \operatorname{diam} A-\operatorname{diam} H$. Suppose, on the contrary, that $\operatorname{dist}(A, H)>$ $\operatorname{diam} A-\operatorname{diam} H=\gamma$ and denote by $f, g: K \rightarrow \mathbb{R}$ the lower and upper semicontinuous functions, respectively, satisfying $H=[f, g]$ and $g(t)-f(t)=\operatorname{diam} H$ for every $t \in \mathcal{D}_{f} \cap \mathcal{D}_{g}$. Then the set

$$
H+\gamma B=[f, g]+[-\gamma, \gamma]=[f-\gamma, g+\gamma]
$$

does not contain $A$. (The last equality follows from Proposition 2.3 in [13], and $\gamma$ denotes a constant function.) This means that there is $\varphi \in A$ and $t_{0} \in K$ such that either $\varphi\left(t_{0}\right)>g\left(t_{0}\right)+\gamma$ or $\varphi\left(t_{0}\right)<f\left(t_{0}\right)-\gamma$. The proof is similar in both cases, 
so we may assume that $\varphi\left(t_{0}\right)>g\left(t_{0}\right)+\gamma$. Since $g$ is upper semicontinuous, there is an open neighborhood $U$ of $t_{0}$ such that $\varphi(t)>g(t)$ for every $t \in U$, and, as a consequence, we may assume that $t_{0}$ is a point of continuity of $f$ and $g$. By using Proposition 2.2 in [13, if we fix $0<\varepsilon<\varphi\left(t_{0}\right)-g\left(t_{0}\right)-\gamma$ we can choose $h \in H$ such that $h\left(t_{0}\right)-f\left(t_{0}\right)<\varepsilon$. Then

$$
\begin{aligned}
\operatorname{diam} A \geq\|\varphi-h\|_{\infty} & \geq \varphi\left(t_{0}\right)-h\left(t_{0}\right) \\
& \geq \varphi\left(t_{0}\right)-f\left(t_{0}\right)+\varepsilon \\
& \geq g\left(t_{0}\right)+\gamma-f\left(t_{0}\right)+\varepsilon=\operatorname{diam} A+\varepsilon
\end{aligned}
$$

provides the contradiction we are looking for.

Once we have proved the two claims, the proof of the Lipschitz continuity of $\gamma$ is fairly easy: let $C, D \in \mathcal{H}$ and $\varepsilon>0$ be such that $\operatorname{dist}(C, D) \leq \varepsilon$. If we consider $A \in \gamma(D)$, then $C \subset D+\varepsilon B \subset A+\varepsilon B$. Since $A+\varepsilon B$ is again diametrically maximal, Claim 1 ensures the existence of $H \in \gamma(C)$ such that $H \subset A+\varepsilon B$. Hence

$$
\begin{aligned}
\operatorname{dist}(A, H) & \leq \operatorname{dist}(A, A+\varepsilon B)+\operatorname{dist}(A+\varepsilon B, H) \\
& \leq \varepsilon+\operatorname{diam}(A+\varepsilon B)-\operatorname{diam} H \\
& =\varepsilon+\operatorname{diam} A+2 \varepsilon-\operatorname{diam} H \\
& =\operatorname{diam} D-\operatorname{diam} C+3 \varepsilon,
\end{aligned}
$$

where Claim 2 has been used in second inequality. Changing the roles of $C$ and $D$ in the above argument, we obtain finally that $\operatorname{dist}(\gamma(C), \gamma(D)) \leq \mid \operatorname{diam} D-$ $\operatorname{diam} C \mid+3 \varepsilon \leq 5 \varepsilon$.

It is important to point out that there are spaces failing the property stated in Claim 1, and an easy example is Euclidean spaces (finite-dimensional spaces endowed with the Euclidean norm). Consider, in $\mathbb{R}^{2}$ with the usual norm, the segment $C=\{(x, 0),-1 \leq x \leq 1\}$ and let $D_{n}=(0,-n)+(n+1 / n) B$. Then $\gamma\left(D_{n}\right)=D_{n}$ and $C \subset D_{n}$ for every $n \in \mathbb{N}$. However, for $n$ big enough, $D_{n}$ contains no completion of $C$. It could be interesting to study what Banach spaces satisfy the Claim 1 property. Related to this problem is the following result of Baronti and Papini [1] which generalizes to any Banach space a previous result proved independently by Vrecica [22] and Scott [21] for finite-dimensional spaces: if $C \in \mathcal{H}$ is included in a ball $D$ with radius $r$ satisfying $\operatorname{diam} C / 2 \leq r \leq \operatorname{diam} C$, then there is a completion of $C$ included in $D$.

\section{Continuity of $\gamma$ in Euclidean spaces}

It is a natural question to ask what continuity properties are satisfied by $\gamma$ in Euclidean spaces. Recall that, in this context, diametrically maximal sets always have constant width. The trouble with these spaces is that we have no handy characterizations of $\gamma(C)$, for most $C \in \mathcal{H}$. Actually, the goal of a recent paper by T. Lachand-Robert and E. Oudet [11] is to provide an explicit construction of constant width sets in any Euclidean space.

Theorem 4.1. The complete hull mapping $\gamma$ is continuous in finite-dimensional Euclidean spaces.

Proof. Let $C, D \in \mathcal{H}$ and $\varepsilon>0$ be such that $\operatorname{dist}(C, D)<\varepsilon$. If we consider $A \in$ $\gamma(D)$, then $C \subset D+\varepsilon B \subset A+\varepsilon B$. We can try to repeat the argument of Theorem 3.1 by looking for a completion $H \in \gamma(C)$ such that $H \subset A+\varepsilon B$. However, it may 
well happen that no completion of $C$ is included in $A+\varepsilon B$ : consider in $\left(\mathbb{R}^{2},\|\cdot\|_{2}\right)$ the sets $D=B \cap\left\{(x, y) \in \mathbb{R}^{2}: y \geq 0\right\}$ and $C=(0,2)+\left(B \cap\left\{(x, y) \in \mathbb{R}^{2}: y \leq 0\right\}\right)$, which satisfy $\operatorname{dist}(C, D)=\sqrt{5}-1$. If we define $A=B$, which is a completion of $D$, there is no $H \in \gamma(C)$ included in $A+(\sqrt{5}-1) B=\sqrt{5} B$. Indeed, the point $(0,2-\sqrt{3})$, together with $(1,2)$ and $(-1,2)$, the extreme points of $C$, form an equilateral triangle. By using an argument of elementary geometry we know that $(0,4-\sqrt{3})$ must be in any completion of $C$; however, $(0,4-\sqrt{3}) \notin \sqrt{5} B$.

Going back to the general setting, once we know that $A+\varepsilon B$ might contain no completion of $C$, what can we hope to find inside of $A+\varepsilon B$ ? Consider the family

$$
\mathcal{F}=\left\{F \in \mathcal{H}: C \subset F \subset A+\varepsilon B, \operatorname{diam} F=d_{C}\right\}
$$

endowed with the partial order given by inclusion. Since every totally ordered chain $\left\{F_{i}\right\} \subset \mathcal{F}$ has a maximal element $F_{0}=\bigcup_{i} F_{i} \in \mathcal{F}$, we can apply Zorn's lemma to conclude that there exists a maximal element $H_{0} \in \mathcal{F}$. Though $H_{0}$ is only an auxiliary body, since it need not be diametrically maximal, it satisfies however a useful property that will be used later:

$$
A+\varepsilon B \subset H_{0}+\alpha B,
$$

where $\alpha^{2}=8 d_{C} \varepsilon+16 \varepsilon^{2}$ (and $\alpha>0$ ). Indeed, pick $a \in A+\varepsilon B$ and assume that $a \notin H_{0}$ (otherwise there is nothing to show). There exists $c \in H_{0}$ such that $\|a-c\|=\inf \left\{\|a-z\|: z \in H_{0}\right\}$, and there exists $c^{\prime} \in H_{0}$ satisfying $\left\|c-c^{\prime}\right\|=d_{C}$. If not, $\sup \left\{\|c-z\|: z \in H_{0}\right\}<d_{C}$ would imply

$$
\operatorname{diam}\left(H_{0} \cup(\lambda a+(1-\lambda) c)\right)<d_{C}
$$

for some $\lambda \in(0,1)$, in contradiction to the maximality of $H_{0}$ in $\mathcal{F}$. We can now use the properties of the Euclidean norm to estimate

$$
\begin{aligned}
d_{C}^{2}+\|a-c\|^{2} & =\left\|c-c^{\prime}\right\|^{2}+\|a-c\|^{2} \\
& \leq\left\|a-c^{\prime}\right\|^{2} \\
& \leq(\operatorname{diam} A+2 \varepsilon)^{2} \leq\left(d_{C}+4 \varepsilon\right)^{2} \\
& =d_{C}^{2}+8 d_{C} \varepsilon+16 \varepsilon^{2} ;
\end{aligned}
$$

hence $\|a-c\| \leq \alpha$, proving (4.1). However, as we already mentioned, we cannot ensure that $H_{0} \in \gamma(C)$. To solve this problem, let $\beta^{2}=2\left(d_{C}+4 \varepsilon\right) \alpha$ (with $\beta>0$ ) and define the family

$$
\mathcal{G}=\left\{G \in \mathcal{H}: H_{0} \subset G \subset A+(\varepsilon+\beta) B, \operatorname{diam} G=d_{C}\right\},
$$

endowed again with the partial order given by the inclusion, and again apply Zorn's lemma to obtain a maximal element $H \in \mathcal{G}$. We claim that $H$ is diametrically maximal, and then, as a consequence, we obtain $H \in \gamma(C)$. Indeed, consider $y \notin H$. If $y \in A+(\varepsilon+\beta) B$ it is clear that $\operatorname{diam}(H \cup\{y\})>\operatorname{diam} H$ because of the maximality of $H$ in $\mathcal{G}$. Suppose that $y \notin A+(\varepsilon+\beta) B$ and consider a point $y^{\prime} \in A+\varepsilon B$ satisfying $\left\|y-y^{\prime}\right\|=\inf \{\|y-z\|: z \in A+\varepsilon B\}$. Notice that

$$
\left\|y-y^{\prime}\right\|^{2}>\beta=4 \sqrt{2}\left(d_{C}+4 \varepsilon\right) \sqrt{d_{C}+2 \varepsilon} \sqrt{\varepsilon} .
$$

Let $y^{\prime \prime}$ be a diametral point of $y^{\prime}$ in $A+\varepsilon B$, namely $\left\|y^{\prime}-y^{\prime \prime}\right\|=\operatorname{diam} A+2 \varepsilon$, and let $z_{0} \in H_{0}$ satisfy $\left\|z_{0}-y^{\prime \prime}\right\| \leq \alpha$ (here we use (4.1)). Then

$$
\begin{aligned}
\left\|y-z_{0}\right\|^{2} & \geq\left\|y-y^{\prime}\right\|^{2}+\left\|y^{\prime}-z_{0}\right\|^{2} \\
& \geq\left\|y-y^{\prime}\right\|^{2}+\left(\left\|y^{\prime}-y^{\prime \prime}\right\|-\left\|y^{\prime \prime}-z_{0}\right\|\right)^{2}
\end{aligned}
$$




$$
\begin{aligned}
& \geq\left\|y-y^{\prime}\right\|^{2}+\left\|y^{\prime}-y^{\prime \prime}\right\|^{2}-2\left\|y^{\prime}-y^{\prime \prime}\right\|\left\|y^{\prime \prime}-z_{0}\right\| \\
& \geq\left\|y-y^{\prime}\right\|^{2}+\left(d_{A}+2 \varepsilon\right) 2-2\left\|y^{\prime}-y^{\prime \prime}\right\|\left\|y^{\prime \prime}-z_{0}\right\| \\
& \geq\left\|y-y^{\prime}\right\|^{2}+d_{C}^{2}-2\left\|y^{\prime}-y^{\prime \prime}\right\|\left\|y^{\prime \prime}-z_{0}\right\| \\
& =\left\|y-y^{\prime}\right\|^{2}+d_{C}^{2}-2\left(d_{A}+2 \varepsilon\right)\left\|y^{\prime \prime}-z_{0}\right\| \\
& \geq\left\|y-y^{\prime}\right\|^{2}+d_{C}^{2}-2\left(d_{C}+4 \varepsilon\right)\left\|y^{\prime \prime}-z_{0}\right\| \\
& \geq\left\|y-y^{\prime}\right\|^{2}+d_{C}^{2}-\beta>d_{C}^{2},
\end{aligned}
$$

showing that $H$ is diametrically maximal and therefore $H \in \gamma(C)$, as claimed. As a consequence, using (4.1) and (4.2), we get

$$
\operatorname{dist}(A, H) \leq \max \{\alpha, \varepsilon+\beta\} .
$$

In order to estimate $\operatorname{dist}(\gamma(C), \gamma(D))$, we now fix $H^{\prime} \in \gamma(C)$. By using the same arguments as before, changing the roles of $C$ and $D$, we obtain $A^{\prime} \in \gamma(C)$ satisfying a similar inequality replacing $A, H$ by $A^{\prime}, H^{\prime}$ and $d_{C}$ by $d_{D}$ in the definitions of $\alpha$ and $\beta$. As a consequence, we have the estimate

$$
\operatorname{dist}(\gamma(C), \gamma(D)) \leq \max \left\{\alpha^{\prime}, \varepsilon+\beta^{\prime}\right\},
$$

where $\alpha^{\prime} \geq \alpha$ and $\beta^{\prime} \geq \beta$ are obtained from $\alpha$ and $\beta$ by simply replacing $d_{C}$ and $d_{D}$ by their maximum $d_{C D}=\max \left\{d_{C}, d_{D}\right\}$. Taking into account the definition of $\alpha^{\prime}$ and $\beta^{\prime}$, the above inequality proves the Hölder continuity of $\gamma$. Having in mind that $d_{C D} \leq d_{C}+2 \varepsilon$ we can get an expression showing the continuity of $\gamma$ in $C$.

For small $\varepsilon$, depending on the diameter of the sets, we will obtain a better estimate, namely that $\gamma$ is Hölder continuous of order $1 / 2$; see (4.7). To this end, again fix $C \in \mathcal{H}$ and consider $H \in \gamma(C)$. Since $H$ is a constant width set, the insphere and circumsphere are concentric and their radii, $r_{H}$ and $R_{H}$ respectively, satisfy, on the one hand, $r_{H}+R_{H}=d_{C}$, and, on the other,

$$
d_{C}\left(1-\sqrt{\frac{n}{2 n+2}}\right) \leq r_{H} \leq R_{H} \leq d_{C} \sqrt{\frac{n}{2 n+2}},
$$

where $d_{C}$ denotes, as before, the diameter of $C$ and $n$ is the dimension of the space (for these and other details concerning constant width sets, the reader is referred to the authoritative survey [5]). If we consider the inner parallel body $H \sim \lambda B=\{x \in H: x+\lambda B \subset H\}$, then $r_{H}$ is the greatest number $\lambda$ for which $H \sim \lambda B$ is not empty. The above estimate (4.3) provides a lower bound for the constant $r(C)=\inf \left\{r_{H}: H \in \gamma(C)\right\}$ as well as an upper bound for the constant $R(C)=\sup \left\{R_{H}: H \in \gamma(C)\right\}$, both together implying that

$$
\sup \left\{\frac{R(C)}{r(C)}: C \in \mathcal{H}\right\} \leq \frac{1}{\sqrt{\frac{2 n+2}{n}}-1} \leq \frac{1}{\sqrt{2}-1} .
$$

Consider $\varepsilon<0.42 r(C)$ and $D \in \mathcal{H}$ such that $\operatorname{dist}(C, D) \leq \varepsilon$. To estimate $\operatorname{dist}(\gamma(C), \gamma(D))$ we first prove the inequality

$$
\varepsilon<\min \{r(C), r(D)\}
$$

that will be used later. Obviously, it is clear that $\varepsilon<r(C)$; also,

$$
\begin{aligned}
r(D) & \geq\left(1-\sqrt{\frac{n}{2 n+2}}\right) d_{D} \geq \frac{2-\sqrt{2}}{2} d_{D} \geq \frac{2-\sqrt{2}}{2}\left(d_{C}-2 \varepsilon\right) \\
& \geq \frac{2-\sqrt{2}}{2} d_{C}-(2-\sqrt{2}) \varepsilon \geq \frac{2-\sqrt{2}}{2} r(C) \frac{\sqrt{2 n+2}}{\sqrt{n}}-(2-\sqrt{2}) \varepsilon
\end{aligned}
$$




$$
\begin{aligned}
& \geq \frac{2-\sqrt{2}}{2} r(C) \sqrt{2}-\frac{2-\sqrt{2}}{2} r(C) \geq \frac{2-\sqrt{2}}{2} r(C)(\sqrt{2}-1) \\
& \geq 0.42 r(C)>\varepsilon .
\end{aligned}
$$

We now begin by defining the family

$$
\mathcal{F}=\left\{F \in \mathcal{H}: D \cup(H \sim \varepsilon B) \subset F \subset H+\varepsilon B, \operatorname{diam} F=\operatorname{diam} D=d_{D}\right\},
$$

which is nonempty since conv $(D \cup(H \sim \varepsilon B)) \in \mathcal{F}$. Indeed, $D \subset C+\varepsilon B \subset H+\varepsilon B$, hence $\operatorname{conv}(D \cup(H \sim \varepsilon B)) \subset H+\varepsilon B$, and we just need to check that

$$
\operatorname{diam}(D \cup(H \sim \varepsilon B)) \leq d_{D} .
$$

On the one hand,

$$
\operatorname{diam}(H \sim \varepsilon B) \leq \operatorname{diam} H-2 \varepsilon=d_{C}-2 \varepsilon \leq d_{D} .
$$

On the other, if $h \in H \sim \varepsilon B$ and $g \in D$, there is $c \in C$ such that $\|g-c\| \leq \varepsilon$, and so

$$
\|h-g\| \leq\|h-c\|+\|c-g\| \leq d_{D}-\varepsilon+\varepsilon<d_{D} .
$$

Now $\mathcal{F}$ is again endowed with the partial order given by the inclusion and, by the arguments already mentioned before, there is a maximal element $A_{0} \in \mathcal{F}$. Notice that the convex body $A_{0}$ satisfies

$$
H \subset(H \sim \varepsilon B)+\frac{R_{H}}{r_{H}} \varepsilon B \subset A_{0}+\frac{R_{H}}{r_{H}} \varepsilon B \subset A_{0}+\frac{R(C)}{r(C)} \varepsilon B .
$$

The first inclusion is due to Thales' theorem (given an arbitrary point of $H$, consider the segment $S$ joining this point with the incenter $x_{H}$, the ball with center in $x_{H}$ and radius $r_{H}$, and finally a suitable ball with center in $S$ and radius $3 \varepsilon$ ), while the third inclusion is due to the definitions of $r(C)$ and $R(C)$, respectively. However, $A_{0}$ need not be diametrically maximal, and thus we cannot say that $A_{0} \in \gamma(D)$. We define a new family

$$
\mathcal{G}=\left\{G \in \mathcal{H}: A_{0} \subset G \subset H+\left(\sqrt{\varepsilon}+\sqrt{2} \sqrt{d_{C}+2 \varepsilon} \sqrt{\frac{R(C)}{r(C)}}\right) \sqrt{\varepsilon} B, \operatorname{diam} F=d_{D}\right\}
$$

which is nonempty ( $A_{0} \in \mathcal{G}$, for instance) and is endowed again with the partial order given by the inclusion. Again applying Zorn's lemma, we obtain a maximal element $A$. We claim that $A$ is diametrically maximal and, consequently, $A \in \gamma(D)$. To prove the claim, consider $y \notin A$. If $y \in H+\left(\sqrt{\varepsilon}+\sqrt{2} \sqrt{d_{C}+2 \varepsilon} \sqrt{\frac{R(C)}{r(C)}}\right) \sqrt{\varepsilon} B$ it is clear that $\operatorname{diam}(A \cup\{y\})>\operatorname{diam} A$ because of the maximality of $A$ in $\mathcal{G}$. Suppose that

$$
y \notin H+\left(\sqrt{\varepsilon}+\sqrt{2} \sqrt{d_{C}+2 \varepsilon} \sqrt{\frac{R(C)}{r(C)}}\right) \sqrt{\varepsilon} B,
$$

denote by $y^{\prime}$ the nearest point of $y$ in $H+\varepsilon B$ and let $y^{\prime \prime}$ be a diametral point of $y^{\prime}$ in $H+\varepsilon B$; that is, $\left\|y^{\prime}-y^{\prime \prime}\right\|=\operatorname{diam} H+2 \varepsilon$. If we consider $z_{0} \in A_{0}$ satisfying $\left\|z_{0}-y^{\prime \prime}\right\| \leq \frac{R(C)}{r(C)} \varepsilon$, then

$$
\begin{aligned}
\left\|y-z_{0}\right\|^{2} & \geq\left\|y-y^{\prime}\right\|^{2}+\left\|y^{\prime}-z_{0}\right\|^{2} \\
& \geq\left\|y-y^{\prime}\right\|^{2}+\left(\left\|y^{\prime}-y^{\prime \prime}\right\|-\left\|y^{\prime \prime}-z_{0}\right\|\right)^{2} \\
& \geq\left\|y-y^{\prime}\right\|^{2}+\left\|y^{\prime}-y^{\prime \prime}\right\|^{2}-2\left\|y^{\prime}-y^{\prime \prime}\right\|\left\|y^{\prime \prime}-z_{0}\right\|
\end{aligned}
$$




$$
\begin{aligned}
& \geq\left\|y-y^{\prime}\right\|^{2}+\left(d_{C}+2 \varepsilon\right) 2-2\left(d_{C}+2 \varepsilon\right) \frac{R(C)}{r(C)} \varepsilon \\
& \geq\left\|y-y^{\prime}\right\|^{2}+d_{D} 2-2\left(d_{C}+2 \varepsilon\right) \frac{R(C)}{r(C)} \varepsilon \\
& >d_{C}^{2} ;
\end{aligned}
$$

hence $A$ is diametrically maximal and $A \in \gamma(D)$, as claimed. Now, in virtue of (4.6) and the definition of $\mathcal{G}$, we get

$$
\begin{aligned}
\operatorname{dist}(A, H) & \leq \max \left\{\frac{R(C)}{r(C)} 3 \varepsilon, \varepsilon+\sqrt{2} \sqrt{d_{C}+2 \varepsilon} \sqrt{\frac{R(C)}{r(C)}} \sqrt{\varepsilon}\right\} \\
& \leq \max \left\{\frac{3 \varepsilon}{\sqrt{2}-1}, \varepsilon+\sqrt{2} \sqrt{\frac{d_{C}+2 \varepsilon}{\sqrt{2}-1}} \sqrt{\varepsilon}\right\} \\
& \leq \max \left\{2,42 \varepsilon, \varepsilon+2,22 \sqrt{d_{C}+2 \varepsilon} \sqrt{\varepsilon}\right\} .
\end{aligned}
$$

Again, in order to estimate $\operatorname{dist}(\gamma(C), \gamma(D))$, we now fix in turn a completion $A^{\prime} \in \gamma(D)$. Then, using the fact that $\varepsilon<r(D) / 3$ (see 4.5) and the above arguments, we can find $H^{\prime} \in \gamma(C)$ such that

$$
\operatorname{dist}\left(H^{\prime}, A^{\prime}\right) \leq \max \left\{\frac{\varepsilon}{\sqrt{2}-1}, \varepsilon+\sqrt{2} \sqrt{\frac{d_{D}+2 \varepsilon}{\sqrt{2}-1}} \sqrt{\varepsilon}\right\},
$$

which together with the previous inequality shows that

$$
\begin{aligned}
\operatorname{dist}(\gamma(C), \gamma(D)) & \leq \max \left\{\frac{\varepsilon}{\sqrt{2}-1}, \varepsilon+\sqrt{2} \sqrt{\frac{d_{C D}+2 \varepsilon}{\sqrt{2}-1}} \sqrt{\varepsilon}\right\} \\
& \leq \max \left\{\frac{\varepsilon}{\sqrt{2}-1}, \varepsilon+\sqrt{2} \sqrt{\frac{d_{C}+4 \varepsilon}{\sqrt{2}-1}} \sqrt{\varepsilon}\right\}
\end{aligned}
$$

for every $D \in \mathcal{H}$ such that $\operatorname{dist}(C, D) \leq \varepsilon \leq 0.42 r(C)$. It is not difficult to check that, with the same arguments, we can prove the following result: for every $C, D \in \mathcal{H}$ satisfying $\operatorname{dist}(C, D)=\varepsilon<\min \left\{d_{C}, d_{D}\right\}(2-\sqrt{2}) / 2$, we have the estimate (4.7). The reason is that

$$
\min \left\{d_{C}, d_{D}\right\} \frac{2-\sqrt{2}}{2} \leq \min \{r(C), r(D)\},
$$

and the proof can then follow from (4.5). Finally, a simple estimate of the real numbers appearing in (4.7) shows (1.1).

Though the above theorem shows the continuity of $\gamma$ in Euclidean spaces (it is indeed locally Hölder continuous), we don't know whether it is actually Lipschitz. The technique used in the proof, based mainly in Zorn's lemma, does not give enough control on the completions. The question remains open as to whether there is a Banach space where $\gamma$ is not continuous. A good candidate is the threedimensional space considered by Boltyanski, Martini and Soltan in [4 that was later used in [17] to prove that the ball hull mapping need not be continuous. 


\section{REFERENCES}

[1] M. Baronti and P. L. Papini, Diameters, centers and diametrically maximal sets, Rend. Circolo Mat. Palermo Suppl. (II) 38 (1995), 11-24. MR1346789 (96f:52009)

[2] E. Behrends, and P. Harmand, Banach spaces which are proper M-ideals, Studia Math. 81 (1985), 159-169. MR818178 (87f:46031)

[3] E. Behrends, Points of symmetry of convex sets in the two-dimensional complex space-a counterexample to D. Yost's problem. Math. Ann. 290 (1991), no. 3, 463-471. MR.1116232 (92f:46007)

[4] V. G. Boltyanski, H. Martini and P. S. Soltan, Excursions into Combinatorial Geometry, Springer, Berlin, 1997. MR 1439963 (98b:52001)

[5] G. D. Chakerian and H. Groemer, Convex bodies of constant width, in Convexity and its Applications, P. Gruber and J. Wills, Eds., Birkhäuser, 1983, 49-96. MR.731106 (85f:52001)

[6] H. G. Eggleston, Sets of constant width in finite-dimensional Banach spaces, Israel J. Math. 3 (1965), 163-172. MR0200695 (34:583)

[7] L. Euler, De Curvis Triangularibus, Acta Academiae Scientarum Imperialis Petropolitinae (1778), 3-30. Opera Omnia: Series 1, Volume 28, pp. 298-321.

[8] C. Franchetti, Relationship between the Jung constant and a certain projection constant in Banach spaces, Ann. Univ. Ferrara, N. Ser. 23 (1977), 39-44. MR0493263 (58:12292)

[9] H. Groemer, On complete convex bodies, Geom. Dedicata 20 (1986), 319-334. MR845426 (87h:52008)

[10] E. Heil and H. Martini, Special convex bodies, in Handbook of Convex Geometry, Vol. A, P. Gruber and J. Wills, Eds., North-Holland, 1993, 347-385. MR.1242985 (94h:52001)

[11] T. Lachand-Robert and E. Oudet, Bodies of constant width in arbitrary dimensions, Math. Nach. 280 (7) (2007), 740-750. MR2321138 (2008d:52002)

[12] E. Meissner, Über Punktmengen konstanter Breite, Vierteljahresschr. naturforsch. Ges Zürich 56 (1911), 42-50. Jbuch. 42, p. 91.

[13] J. P. Moreno, Semicontinuous functions and convex sets in $C(K)$ spaces, J. Austral. Math. Soc. 82 (2007), 111-121. MR2301973 (2008m:46036)

[14] J. P. Moreno, Porosity and diametrically maximal sets in $C(K)$, Monatsh. Math. $152(2007)$, 255-263. MR.2357520 (2008k:46050)

[15] J. P. Moreno, P. L. Papini and R. R. Phelps, Diametrically maximal and constant width sets in Banach spaces, Canad. J. Math. 58 (4) (2006), 820-842. MR2245275 (2007j:52003)

[16] J. P. Moreno, P. L. Papini and R. R. Phelps, New families of convex sets related to diametral maximality, J. Convex. Anal. 13 (2006), 823-837. MR2291568(2008c:52001)

[17] J. P. Moreno and R. Schneider, Continuity properties of the ball hull mapping, Nonlinear Anal. 66 (2007), 914-925. MR2288440 (2007m:46026)

[18] R. Paya and A. Rodríguez-Palacios, Banach spaces which are semi-L-summands in their biduals, Math. Ann. 289 (1991), 529-542. MR.1096186 (92e:46024)

[19] A. Rodríguez-Palacios, Infinite-dimensional sets of constant width and their applications, Extracta Math. Actas del II Congreso de Análisis Funcional, Jarandilla, España (1990), 140-151.

[20] A. Rodríguez-Palacios, Properly semi-L-embedded complex spaces, Studia Math. 106 (1993), 197-202. MR.1240314 (94k:46025)

[21] P. R. Scott, Sets of constant width and inequalities, Quart. J. Math. Oxford Ser. (2) 32 (1981), no. 127, 345-348. MR625646 (82k:52013)

[22] S. Vrećica, A note on sets of constant width, Publ. Inst. Math. (Beograd) (N.S.) 29 (43) (1981), 289-291. MR657118 (83g:52006)

Departamento Matemáticas, Facultad de Ciencias, Universidad Autónoma de Madrid, MADRID 28049, SPAIN

E-mail address: josepedro.moreno@uam.es 